\title{
On Abstract Economies and Their Applications
}

\author{
Chien-Hao Huang and Liang-Ju Chu \\ Department of Mathematics, National Taiwan Normal University, No. 88, Section 4, Ting-Chou Road, Taipei 116, Taiwan \\ Correspondence should be addressed to Liang-Ju Chu; chulj@math.ntnu.edu.tw
}

Received 10 July 2013; Accepted 22 August 2013

Academic Editor: Wei-Shih Du

Copyright (c) 2013 C.-H. Huang and L.-J. Chu. This is an open access article distributed under the Creative Commons Attribution License, which permits unrestricted use, distribution, and reproduction in any medium, provided the original work is properly cited.

We establish a new equilibrium existence theorem of generalized abstract economies with general preference correspondences. As an application, we derive an existence theorem of generalized quasi-variational inequalities in the general setting of $l$.c.-spaces without any linear structure.

\section{Introduction and Preliminary}

Let $I$ be any (finite or infinite) set of agents. A generalized abstract economy is defined as a family of order quintuples $\boldsymbol{\Omega}=\left(X_{\alpha}, A_{\alpha}, B_{\alpha}, F_{\alpha}, P_{\alpha}\right)_{\alpha \in I}$ with $X:=\prod_{\alpha \in I} X_{\alpha}$ such that for each $\alpha \in I, X_{\alpha}$ is a topological space, $A_{\alpha}, B_{\alpha}: X \rightarrow 2^{X_{\alpha}}$ are constraint correspondences, $F_{\alpha}: X \rightarrow 2^{X_{\alpha}}$ is a fuzzy constraint correspondence, and $P_{\alpha}: X \times X \rightarrow 2^{X_{\alpha}}$ is a preference correspondence. In a real market, any preference of a real agent would be unstable by the fuzziness of consumers' behavior or market situations. Thus, it is reasonable to introduce fuzzy constraint correspondences in defining an abstract economy. An equilibrium point of $\boldsymbol{\Omega}$ is a point $(\widehat{x}, \hat{y}) \in X \times X$ such that for each $\alpha \in I, \hat{x}_{\alpha} \in \operatorname{cl} B_{\alpha}(\widehat{x}), \hat{y}_{\alpha} \in$ $F_{\alpha}(\widehat{x})$, and $A_{\alpha}(\widehat{x}) \cap P_{\alpha}(\widehat{x}, \widehat{y})=\emptyset$, where $\widehat{x}_{\alpha}$ and $\hat{y}_{\alpha}$ denote the projections of $\hat{x}$ and $\hat{y}$ from $X$ to $X_{\alpha}$, respectively.

In case $F_{\alpha}(x)=X_{\alpha}$ for each $x \in X$ and $P_{\alpha}$ is independent of the second variable, that is, $P_{\alpha}: X \rightarrow 2^{X_{\alpha}}$, the above generalized abstract economy reduces to the standard abstract economy $\boldsymbol{\Omega}_{\mathrm{s}}:=\left(X_{\alpha}, A_{\alpha}, B_{\alpha}, P_{\alpha}\right)_{\alpha \in I}$, in which an equilibrium point of $\boldsymbol{\Omega}_{\mathrm{s}}$ is a point $\widehat{x} \in X$ such that for each $\alpha \in I, \hat{x}_{\alpha} \in$ $\operatorname{cl} B_{\alpha}(\widehat{x})$ and $A_{\alpha}(\widehat{x}) \cap P_{\alpha}(\widehat{x})=\emptyset$. When $A_{\alpha}=B_{\alpha}$ and each $X_{\alpha}$ is a topological vector space, the standard abstract economy $\boldsymbol{\Omega}_{\mathbf{s}}$ coincides with the classical definition of Shafer and Sonnenschein [1]. For more details on abstract economies, see, for example, [2-14] and the references therein.

Throughout this paper, all topological spaces are assumed to be Hausdorff. In order to establish our main results, we first give some basic notations. For a nonempty set $C$ of a topological space $X$, we denote the set of all subsets of $C$ by $2^{C}$, the set of all nonempty finite subsets of $C$ by $\langle X\rangle$, the interior of $C$ by int $C$, and the closure of $C$ by $\operatorname{cl} C$.

Let $\left\{\Gamma_{D}\right\}$ be a family of some nonempty contractible subsets of a topological space $X$ indexed by $D \in\langle X\rangle$ such that $\Gamma_{D} \subset \Gamma_{D^{\prime}}$ whenever $D \subset D^{\prime}$. The pair $\left(X,\left\{\Gamma_{D}\right\}\right)$ is called an $H$-space. Given an $H$-space $\left(X,\left\{\Gamma_{D}\right\}\right)$, a nonempty subset $C$ of $X$ is said to be $H$-convex if $\Gamma_{D} \subseteq C$ for all $D \in\langle C\rangle$. For a nonempty subset $C$ of $X$, we define the $H$-convex hull of $C$ as

$$
H \text {-coC := }\{\{K \mid K \text { is } H \text {-convex in } X \text { and } C \subseteq K\} \text {. }
$$

It is known that if $x \in H$-coC, then there exists a finit subset $D$ of $C$ such that $x \in H$-coD. Moreover, for any $D \in\langle X\rangle$, $H$-co $D$ is called a polytope. We will say that $X$ is an $H$-space with precompact polytopes if any polytope of $X$ is precompact. For example, a locally convex topological vector space $X$ is an $H$-space with precompact polytopes, by setting $\Gamma_{D}=$ co $D$ for all $D \in\langle X\rangle$.

An $H$-space $\left(X,\left\{\Gamma_{D}\right\}\right)$ is called an l.c.-space if $X$ is a uniform space whose topology is induced by its uniformity $\mathcal{U}$, and there is a base $\mathscr{B}$ consisting of symmetric entourages in $\mathcal{U}$ such that for each $V \in \mathscr{B}$, the set $V(E):=\{y \in X \mid(x, y) \in V$ for some $x \in E\}$ is $H$-convex whenever $E$ is $H$-convex. We will use the notation $(X, \mathscr{U}, \mathscr{B})$ to stand for an l.c.-space. For details of uniform spaces, we refer to [15]. In a recent 
paper [16], we introduce a new measure of precompactness of a subset $A$ in an l.c.-space $(X, \mathcal{U}, \mathscr{B})$ by

$$
\begin{aligned}
Q(A):= & \{V \in \mathscr{B} \mid A \subseteq \mathrm{cl}(V(K)) \\
& \text { for some precompact set } K \text { of } X\} .
\end{aligned}
$$

Let $\left(X_{\alpha}, \mathscr{U}_{\alpha}, \mathscr{B}_{\alpha}\right)_{\alpha \in I}$ be a family of l.c.-spaces with precompact polytopes, where $I$ is a finite or infinite index set and $X=\prod_{\alpha \in I} X_{\alpha}$. For each $\alpha \in I$, let $\pi_{\alpha}$ be the projection of $X$ onto $X_{\alpha}$ and $Q_{\alpha}$ a measure of precompactness in $X_{\alpha}$. We say that a set-valued mapping $T_{\alpha}: X \rightarrow 2^{X_{\alpha}}$ is $Q_{\alpha^{-}}$ condensing if $Q_{\alpha}\left(\pi_{\alpha}(C)\right) \subsetneq Q_{\alpha}\left(T_{\alpha}(C)\right)$ for every $C$ satisfying $\pi_{\alpha}(C)$ is a nonprecompact subset of $X_{\alpha}$. It is clear that for any set-valued mapping $T: X \rightarrow 2^{Y}$ and any measure $Q$ in $Y, T$ is $Q$-condensing whenever $Y$ is compact.

Let $X$ be a topological space, let $Y$ be an $H$-space, and let $S, T: X \rightarrow 2^{Y}$ be two set-valued mappings.

(1) $T$ is said to be upper semicontinuous (u.s.c.) if for each $x \in X$ and each open subset $V$ of $Y$ with $T(x) \subseteq V$, there exists a neighborhood $N_{x}$ of $x$ such that $T(z) \subseteq$ $V$ for all $z \in N_{x}$.

(2) $T$ is said to be transfer open valued on $X$ if for each $x \in X$, for each $y \in T(x)$, there exists some $x^{\prime} \in X$ such that $y \in \operatorname{int} T\left(x^{\prime}\right)$.

(3) $T$ is said to be transfer open inverse valued in $Y$ if $T^{-1}$ is transfer open valued on $Y$, where $T^{-1}: Y \rightarrow 2^{X}$ is defined by

$$
T^{-1}(y):=\{x \in X \mid y \in T(x)\} \quad \forall y \in Y .
$$

(4) The set-valued mappings $S \cap T: X \rightarrow 2^{Y}$ and $\operatorname{cl} T$ : $X \rightarrow 2^{Y}$ are defined by

$$
\begin{array}{r}
(S \cap T)(x):=S(x) \cap T(x), \\
\operatorname{cl} T(x):=\operatorname{cl}(T(x)), \quad \forall x \in X .
\end{array}
$$

Further, we denote by $\mathbb{H}(X, Y)$ the class of all u.s.c. setvalued mappings $T: X \rightarrow 2^{Y}$ with nonempty closed $H$-convex values.

\section{Main Results}

The following fundamental theorems will play an important role in proving our main theorem.

Theorem A (see [16]). Let $\left(X_{\alpha}, \mathscr{U}_{\alpha}, \mathscr{B}_{\alpha}\right)_{\alpha \in I}$ be a family of l.c.spaces with precompact polytopes, $X:=\prod_{\alpha \in I} X_{\alpha}$, and let $T_{\alpha}$ : $X \rightarrow 2^{X_{\alpha}}$ be $Q_{\alpha}$-condensing. Then there exists a nonempty compact $H$-convex subset $K:=\prod_{\alpha \in I} K_{\alpha}$ of $X$ such that $T_{\alpha}(K) \subseteq K_{\alpha}$.

Theorem B (see [16]). Let $\left(X_{\alpha}, \mathscr{U}_{\alpha}, \mathscr{B}_{\alpha}\right)_{\alpha \in I}$ be a family of l.c.spaces with precompact polytopes and $X:=\prod_{\alpha \in I} X_{\alpha}$. If $T_{\alpha}$ : $X \rightarrow 2^{X_{\alpha}}$ is an u.s.c. $Q_{\alpha}$-condensing mapping with closed $H$ convex values for each $\alpha \in I$, then $T:=\prod_{\alpha \in I} T_{\alpha}$ has a fixed point. lows.

Next, we list and establish some essential lemmas as fol-

Lemma 1 (see [12]). If $X$ is an l.c.-space and $E$ is an $H$-convex subset of $X$, then $\mathrm{cl} E$ is also $H$-convex.

Lemma 2 (see [12]). Let $X$ be a topological space and let $\left(Y,\left\{\Gamma_{D}\right\}\right)$ be a compact l.c.-space. If $T: X \rightarrow 2^{Y}$ is an u.s.c. set-valued mapping, then the mapping $x \mapsto \mathrm{cl}[H$-coT $(x)]$ is also u.s.c. with compact $H$-convex values.

Lemma 3 (see [7]). Let $X$ and $Y$ be topological spaces and let $S: X \rightarrow 2^{Y}$ be a transfer open valued mapping. Then $\bigcup_{x \in X} S(x)=Y \backslash\left(\cap_{x \in X} \operatorname{cl}(Y \backslash S(x))\right)$ and hence $\bigcup_{x \in X} S(x)$ is open in $Y$.

Lemma 4. Let $X$ be paracompact, $\left(Y,\left\{\Gamma_{D}\right\}\right)$ an $H$-space, and $S, T: X \rightarrow 2^{Y}$ be two set-valued mappings such that

(1) $S(x) \neq \emptyset$ and $H-\operatorname{coS}(x) \subseteq T(x)$ for each $x \in X$,

(2) $S$ is transfer open inverse valued in $Y$.

Then $T$ has a continuous selection; that is, there exists a continuous function $f: X \rightarrow Y$ such that $f(x) \in T(x)$ for each $x \in X$.

Proof. Since for each $x \in X, S(x) \neq \emptyset$, it follows that $x \in$ $S^{-1}(y)$ for some $y \in Y$. Since $S$ is transfer open inverse valued in $Y$, there exists some $y_{x} \in Y$ such that $x \in \operatorname{int} S^{-1}\left(y_{x}\right)$. This yields that $\left\{\operatorname{int} S^{-1}(y) \mid y \in Y\right\}$ forms an open cover of $X$. Since $X$ is paracompact, there exists a locally finite open cover $\left\{U_{y} \mid y \in Y\right\}$ such that $U_{y} \subseteq \operatorname{int} S^{-1}(y)$ for each $y \in Y$. By [17, Theorem 3.1], there exists a continuous function $f: X \rightarrow Y$ such that $f(x) \in \Gamma_{\left\{y \in Y \mid x \in U_{y}\right\}}$ for all $x \in X$. Note that for any $x \in X$, there exist finitely many $y \in Y$ such that $x \in U_{y} \subseteq$ $\operatorname{int} S^{-1}(y) \subseteq S^{-1}(y)$. This implies $y \in S(x) \subseteq H$-coS $(x)$, and hence $\left\{y \in Y \mid x \in U_{y}\right\} \in\langle H-\operatorname{coS}(x)\rangle$. It follows that for each $x \in X$, we get

$$
f(x) \in \Gamma_{\left\{y \in Y \mid x \in U_{y}\right\}} \subseteq H-\operatorname{coS}(x) \subseteq T(x) .
$$

Thus, the proof is complete.

We remark that Lemma 4 extends [7, Theorem 2] from topological vector spaces to general $H$-spaces. When $S=$ $T$ and $S$ has open lower sections, Lemma 4 reduces to [18, Theorem 3.1].

Lemma 5. Let $X$ be a compact $H$-space, and let $P: X \times X \rightarrow$ $2^{X}$ be a set-valued mapping such that for each $x \in X, P^{-1}(x)$ is open: then so is $(H-\operatorname{co} P)^{-1}(x)$.

Proof. For each $z_{0} \in X$, we fix an $\left(x_{0}, y_{0}\right) \in(H-\operatorname{co} P)^{-1}\left(z_{0}\right)$. Since $z_{0} \in H-\operatorname{co} P\left(x_{0}, y_{0}\right)$, there is a finite set $\left\{z_{1}, z_{2}, \ldots, z_{n}\right\}$ in $P\left(x_{0}, y_{0}\right)$ such that $z_{0} \in H$-co $\left\{z_{1}, z_{2}, \ldots, z_{n}\right\}$. Since each $P^{-1}\left(z_{i}\right)$ is open, it follows that the set $U:=\bigcap_{i=1}^{n} P^{-1}\left(z_{i}\right)$ is also open and $\left(x_{0}, y_{0}\right) \in U$. To complete the proof, we will show that $U \subseteq(H-\operatorname{co} P)^{-1}\left(z_{0}\right)$. For any $(x, y) \in U$, we have 
$(x, y) \in P^{-1}\left(z_{i}\right)$ for all $i=1,2, \ldots, n$. Accordingly, $z_{i} \in$ $P(x, y)$ for all $i=1,2, \ldots, n$. Hence,

$$
z_{0} \in H-\operatorname{co}\left\{z_{1}, z_{2}, \ldots, z_{n}\right\} \subseteq H-\operatorname{co} P(x, y) .
$$

That is, $(x, y) \in(H-\operatorname{co} P)^{-1}\left(z_{0}\right)$. Consequently, $U \subseteq$ $(H-\operatorname{co} P)^{-1}\left(z_{0}\right)$.

Theorem 6. Let $\mathbf{\Omega}=\left(X_{\alpha}, A_{\alpha}, B_{\alpha}, F_{\alpha}, P_{\alpha}\right)_{\alpha \in I}$ be a generalized abstract economy, where I is a set of agents and $X=\prod_{\alpha \in I} X_{\alpha}$ such that for each $\alpha \in I$,

(1) $X_{\alpha}$ is an l.c.-space with precompact polytopes,

(2) $A_{\alpha}(x) \subseteq \operatorname{cl} B_{\alpha}(x)$ for each $x \in X$,

(3) both $\mathrm{cl} B_{\alpha}$ and $F_{\alpha}$ are $Q_{\alpha}$-condensing mappings in $\mathbb{U}\left(X, X_{\alpha}\right)$,

(4) $x_{\alpha} \notin \mathrm{cl}\left(H-\operatorname{co} P_{\alpha}(x, y)\right)$ for each $x, y \in X$,

(5) $A_{\alpha} \cap\left(H-\operatorname{co} P_{\alpha}\right)$ is transfer open inverse valued in $X_{\alpha}$,

(6) $W_{\alpha}:=\left\{(x, y) \in X \times X \mid A_{\alpha}(x) \cap\left(H-\operatorname{co}_{\alpha}(x, y)\right) \neq \emptyset\right\}$ is paracompact.

Then $\boldsymbol{\Omega}$ has an equilibrium point $(\hat{x}, \hat{y}) \in X \times X$.

Proof. For each $\alpha \in I$, we define $\phi_{\alpha}: X \times X \rightarrow 2^{X_{\alpha}}$ by

$$
\phi_{\alpha}(x, y):=A_{\alpha}(x) \cap\left(H-\operatorname{co}_{\alpha}(x, y)\right), \quad \forall(x, y) \in X \times X .
$$

Assume that $W_{\alpha} \neq \emptyset$. Then for each $(x, y) \in W_{\alpha}$, we have some $y_{\alpha} \in \phi_{\alpha}(x, y)$. Equivalently, $(x, y) \in \phi_{\alpha}^{-1}\left(y_{\alpha}\right)$. It follows that $W_{\alpha}=\bigcup_{y_{\alpha} \in X_{\alpha}} \phi_{\alpha}^{-1}\left(y_{\alpha}\right)$. Since each $\phi_{\alpha}$ is transfer open inverse valued in $X_{\alpha}$ by (5), it follows from Lemma 3 that $W_{\alpha}$ is open in $X \times X$.

For $z_{\alpha} \in X_{\alpha}$, if $(x, y) \in \phi_{\alpha}^{-1}\left(z_{\alpha}\right)$, by using (5), we have some $z_{\alpha}^{\prime} \in X_{\alpha}$ such that $(x, y) \in \operatorname{int} \phi_{\alpha}^{-1}\left(z_{\alpha}^{\prime}\right) \subseteq W_{\alpha}$. Thus, the restriction $\left.\phi_{\alpha}\right|_{W_{\alpha}}: W_{\alpha} \rightarrow 2^{X_{\alpha}}$ is transfer open inverse valued in $X_{\alpha}$. Moreover, by (3), each $\left.\phi_{\alpha}\right|_{W_{\alpha}}(x, y)$ is nonempty and $H$-convex. Therefore, by Lemma 4 , there exists a continuous function $f_{\alpha}: W_{\alpha} \rightarrow X_{\alpha}$ such that $\left.f_{\alpha}(x, y) \in \phi_{\alpha}\right|_{W_{\alpha}}(x, y)$ for each $(x, y) \in W_{\alpha}$.

Since $\mathrm{cl} B_{\alpha}$ and $F_{\alpha}$ are $Q_{\alpha}$-condensing, applying Theorem A, we have two nonempty compact $H$-convex subsets $K$ := $\prod_{\alpha \in I} K_{\alpha}$ and $K^{\prime}:=\prod_{\alpha \in I} K_{\alpha}^{\prime}$ of $X$ such that $\operatorname{cl} B_{\alpha}(K) \subseteq K_{\alpha}$ and $F_{\alpha}\left(K^{\prime}\right) \subseteq K_{\alpha}^{\prime}$. Using these notations, we define a set-valued mapping $S_{\alpha}: K \times K^{\prime} \rightarrow 2^{K_{\alpha} \times K_{\alpha}^{\prime}}$ by

$$
S_{\alpha}(x, y)=\left\{\begin{array}{c}
\operatorname{cl}\left(H-\operatorname{co} f_{\alpha}(x, y)\right) \times F_{\alpha}(x), \\
\text { if }(x, y) \in\left(K \times K^{\prime}\right) \cap W_{\alpha}, \\
\operatorname{cl} B_{\alpha}(x) \times F_{\alpha}(x), \\
\quad \text { if }(x, y) \in\left(K \times K^{\prime}\right) \backslash W_{\alpha} .
\end{array}\right.
$$

We will show that $S_{\alpha} \in \mathbb{H}\left(K \times K^{\prime}, K_{\alpha} \times K_{\alpha}^{\prime}\right)$. Let $V_{\alpha}$ be an open subset of $K_{\alpha} \times K_{\alpha}^{\prime}$. Since $\operatorname{cl}\left[H-\operatorname{co} f_{\alpha}(x, y)\right] \subseteq$ $\operatorname{cl}\left[H-\operatorname{co} \phi_{\alpha}(x, y)\right] \subseteq \operatorname{cl} B_{\alpha}(x)$ for each $(x, y) \in W_{\alpha}$, we have

$$
\begin{aligned}
U_{\alpha}= & \left\{(x, y) \in K \times K^{\prime} \mid S_{\alpha}(x, y) \subseteq V_{\alpha}\right\} \\
= & \left\{(x, y) \in\left(K \times K^{\prime}\right) \cap W_{\alpha} \mid \operatorname{cl}\left[H-\operatorname{co} f_{\alpha}(x, y)\right]\right. \\
& \left.\times F_{\alpha}(x) \subseteq V_{\alpha}\right\} \\
\cup & \left\{(x, y) \in\left(K \times K^{\prime}\right) \backslash W_{\alpha} \mid \operatorname{cl} B_{\alpha}(x) \times F_{\alpha}(x) \subseteq V_{\alpha}\right\} \\
= & \left\{(x, y) \in\left(K \times K^{\prime}\right) \cap W_{\alpha} \mid \operatorname{cl}\left[H-\operatorname{co} f_{\alpha}(x, y)\right]\right. \\
& \left.\times F_{\alpha}(x) \subseteq V_{\alpha}\right\} \\
\cup & \left\{(x, y) \in K \times K^{\prime} \mid \operatorname{cl} B_{\alpha}(x) \times F_{\alpha}(x) \subseteq V_{\alpha}\right\} .
\end{aligned}
$$

It follows from Lemma 2 and the upper semicontinuity of $\mathrm{cl} B_{\alpha} \times F_{\alpha}$ that $U_{\alpha}$ is open in $K \times K^{\prime}$. Hence, $S_{\alpha}$ is u.s.c. Further, by (3) and Lemma 1, each $S_{\alpha}(x, y)$ is nonempty, closed, and $H$-convex. Therefore, $S_{\alpha} \in \mathbb{U}\left(K \times K^{\prime}, K_{\alpha} \times K_{\alpha}^{\prime}\right)$.

Next, we define a set-valued mapping $T_{\alpha}: K \times K^{\prime} \rightarrow$ $2^{K_{\alpha} \times K_{\alpha}^{\prime}}$ by

$$
\begin{aligned}
& T_{\alpha}(x, y) \\
& \quad= \begin{cases}S_{\alpha}(x, y), & \text { if }(x, y) \in\left(K \times K^{\prime}\right) \cap W_{\alpha}, \\
\operatorname{cl} B_{\alpha}(x) \times F_{\alpha}(x), & \text { if }(x, y) \in\left(K \times K^{\prime}\right) \backslash W_{\alpha} .\end{cases}
\end{aligned}
$$

Since $K_{\alpha} \times K_{\alpha}^{\prime}$ is compact, each $T_{\alpha}$ is $Q_{\alpha}$-condensing in $\mathbb{U}\left(K \times K^{\prime}, K_{\alpha} \times K_{\alpha}^{\prime}\right)$. Hence, by Theorem B, the set-valued mapping $\prod_{\alpha \in I} T_{\alpha}$ has a fixed point $(\hat{x}, \widehat{y}) \in K \times K^{\prime}$; that is, $\left(\widehat{x}_{\alpha}, \widehat{y}_{\alpha}\right) \in T_{\alpha}(\widehat{x}, \widehat{y})$ for each $\alpha \in I$. If $(\widehat{x}, \widehat{y}) \in W_{\alpha}$, then

$$
\begin{aligned}
\left(\widehat{x}_{\alpha}, \widehat{y}_{\alpha}\right) & \in \operatorname{cl}\left(S_{\alpha}(\hat{x}, \hat{y})\right) \\
& \subseteq \operatorname{cl}\left(B_{\alpha}(x) \cap\left(H-\operatorname{co} P_{\alpha}(\hat{x}, \hat{y})\right)\right) \times F_{\alpha}(\widehat{x}) .
\end{aligned}
$$

Thus, $\hat{x}_{\alpha} \in \operatorname{cl}\left(H-\operatorname{co} P_{\alpha}(\hat{x}, \hat{y})\right)$, which contradicts with (4). Therefore, $(\widehat{x}, \widehat{y}) \notin W_{\alpha}$ and hence $\widehat{x}_{\alpha} \in \operatorname{cl} B_{\alpha}(\widehat{x}), \hat{y}_{\alpha} \in F_{\alpha}(\widehat{x})$, and $A_{\alpha}(\widehat{x}) \cap P_{\alpha}(\widehat{x}, \widehat{y})=\emptyset$ for each $\alpha \in I$. That is, $(\widehat{x}, \widehat{y})$ is an equilibrium of $\boldsymbol{\Omega}$.

Remark that condition (4) of Theorem 6 can be replaced by a milder condition $x_{\alpha} \notin \operatorname{cl}\left(B_{\alpha}(x) \cap H-\operatorname{co} P_{\alpha}(x, y)\right)$ for each $(x, y) \in W_{\alpha}$. Further, when each l.c.-space $\left(X_{\alpha}, \Gamma^{\alpha}\right)$ satisfies $\Gamma_{\left\{x_{\alpha}\right\}}^{\alpha}=\left\{x_{\alpha}\right\}$, condition (4) can be modified by $x_{\alpha} \notin$ $H-\operatorname{co} P_{\alpha}(x, y)$ without affecting the conclusion.

Corollary 7. Let $\boldsymbol{\Omega}=\left(X_{\alpha}, A_{\alpha}, B_{\alpha}, F_{\alpha}, P_{\alpha}\right)_{\alpha \in I}$ be a generalized abstract economy, where $I$ is a set of agents and $X=\prod_{\alpha \in I} X_{\alpha}$ such that for each $\alpha \in I$,

(1) $\left(X_{\alpha}, \Gamma^{\alpha}\right)$ is an l.c.-space with precompact polytopes, and $\Gamma_{\left\{x_{\alpha}\right\}}^{\alpha}=\left\{x_{\alpha}\right\}$ for each $x_{\alpha} \in X_{\alpha}$, 
(2) $A_{\alpha}(x) \subseteq \operatorname{cl} B_{\alpha}(x)$ for each $x \in X$,

(3) both $\mathrm{cl} B_{\alpha}$ and $F_{\alpha}$ are $Q_{\alpha}$-condensing mappings in $\mathbb{H}\left(X, X_{\alpha}\right)$,

(4) $x_{\alpha} \notin H-\operatorname{co} P_{\alpha}(x, y)$ for each $x, y \in X$,

(5) $A_{\alpha} \cap\left(H-\operatorname{co} P_{\alpha}\right)$ is transfer open inverse valued in $X_{\alpha}$,

(6) $W_{\alpha}:=\left\{(x, y) \in X \times X \mid A_{\alpha}(x) \cap\left(H-\operatorname{co} P_{\alpha}(x, y)\right) \neq \emptyset\right\}$ is paracompact.

Then $\Omega$ has an equilibrium point $(\widehat{x}, \widehat{y}) \in X \times X$.

Proof. According to the proof of Theorem 6 and by virtue of the condition $\Gamma_{\left\{x_{\alpha}\right\}}^{\alpha}=\left\{x_{\alpha}\right\}$ for each $x_{\alpha} \in X_{\alpha}$, we obtain $\operatorname{cl}\left(H\right.$-co $\left.f_{\alpha}(x, y)\right)=f_{\alpha}(x, y)$. It follows that the set-valued mapping $S_{\alpha}$ can be defined by

$S_{\alpha}(x, y)= \begin{cases}f_{\alpha}(x, y) \times F_{\alpha}(x), & \text { if }(x, y) \in\left(K \times K^{\prime}\right) \cap W_{\alpha}, \\ \operatorname{cl} B_{\alpha}(x) \times F_{\alpha}(x), & \text { if }(x, y) \in\left(K \times K^{\prime}\right) \backslash W_{\alpha} .\end{cases}$

Thus, by an analogue proof to Theorem 6, we may conclude that $\Omega$ has an equilibrium point.

Following the proof of Theorem 6 by taking $\phi_{\alpha}(x, y):=$ $\operatorname{cl} B_{\alpha}(x) \cap\left(H-\operatorname{co} P_{\alpha}(x, y)\right)$, we may obtain a new version of equilibrium existence theorem as follows.

Corollary 8. Let $\mathbf{\Omega}=\left(X_{\alpha}, A_{\alpha}, B_{\alpha}, F_{\alpha}, P_{\alpha}\right)_{\alpha \in I}$ be a generalized abstract economy, where $I$ is a set of agents and $X=\prod_{\alpha \in I} X_{\alpha}$ such that for each $\alpha \in I$,

(1) $X_{\alpha}$ is an l.c.-space with precompact polytopes,

(2) $A_{\alpha}(x) \subseteq \operatorname{cl} B_{\alpha}(x)$ for each $x \in X$,

(3) both $\mathrm{cl}_{\alpha}$ and $F_{\alpha}$ are $Q_{\alpha}$-condensing mappings in $\mathbb{H}\left(X, X_{\alpha}\right)$,

(4) $x_{\alpha} \notin \operatorname{cl}\left(H-\operatorname{co} P_{\alpha}(x, y)\right)$ for each $x, y \in X$,

(5) $\operatorname{cl} B_{\alpha} \cap\left(H\right.$-co $\left.P_{\alpha}\right)$ is transfer open inverse valued in $X_{\alpha}$,

(6) $W_{\alpha}:=\left\{(x, y) \in X \times X \mid \operatorname{cl} B_{\alpha}(x) \cap\left(H-\operatorname{co}_{\alpha}(x, y)\right) \neq \emptyset\right\}$ is paracompact.

Then $\Omega$ has an equilibrium point $(\hat{x}, \widehat{y}) \in X \times X$.

Notice that Theorem 6 generalizes [7, Kim-Tan, Theorem 2 ], in which they deal with the case of locally convex topological vector spaces under some compactness conditions, and it also improves [19, Wu-Yuan, Theorem 3] in the setting of locally $H$-convex spaces. We also note that if $X$ is metrizable, the set $W_{\alpha}$ is also metrizable and hence is paracompact. Therefore, the assumption (6) of Theorem 6 is automatically satisfied. Furthermore, if each $X_{\alpha}$ is compact, then both $\mathrm{cl} B_{\alpha}$ and $F_{\alpha}$ are obviously $Q_{\alpha}$-condensing. Thus, we have an immediate consequence, which is a generalization of [7, KimTan, Corollary 1] to $H$-spaces.

Corollary 9. Let $\mathbf{\Omega}=\left(X_{\alpha}, A_{\alpha}, B_{\alpha}, F_{\alpha}, P_{\alpha}\right)_{\alpha \in I}$ be a generalized abstract economy, where $I$ is a set of agents such that for each $\alpha \in I$,
(1) $\left(X_{\alpha}, \Gamma^{\alpha}\right)$ is a metrizable compact l.c.-space, and $\Gamma_{\left\{x_{\alpha}\right\}}^{\alpha}=$ $\left\{x_{\alpha}\right\}$ for each $x_{\alpha} \in X_{\alpha}$,

(2) $A_{\alpha}(x) \subseteq \operatorname{cl} B_{\alpha}(x)$ for each $x \in X$,

(3) $\mathrm{cl} B_{\alpha} \in \mathbb{M}\left(X, X_{\alpha}\right)$, and $F_{\alpha} \in \mathbb{H}\left(X, X_{\alpha}\right)$,

(4) $x_{\alpha} \notin H-\operatorname{co} P_{\alpha}(x, y)$ for each $x, y \in X$,

(5) $A_{\alpha} \cap\left(H\right.$-co $\left.P_{\alpha}\right)$ is transfer open inverse valued in $X_{\alpha}$.

Then $\Omega$ has an equilibrium point $(\widehat{x}, \widehat{y}) \in X \times X$.

We note that our main results focus on the setting of general l.c.-spaces without any linear structure; further, the correspondences are not necessarily lower semicontinuous and do not require the usual open lower section assumption, such as the earlier works [3, Theorem 4], [13, Theorem 3 and its Corollary], [19, Theorems 1 and 3], and [18, Theorem 6.1]. In fact, we can give a simple example applicable for Corollary 9 , while previous results do not.

Example 10. Consider the set $I$ of agents is singleton. Let $X=$ $[0,1]$ and the correspondences $A, B, F: X \rightarrow 2^{X}$ be defined by $A(x)=B(x)=[0,1]$, and $F(x)=\{x\}$ for each $x \in X$. The preference correspondence $P: X \times X \rightarrow 2^{X}$ is defined as follows:

$$
P\left(x_{1}, x_{2}\right)= \begin{cases}\left(\frac{x_{1}+x_{2}}{2}, 1\right], & \text { if } x_{1}<x_{2}, x_{1}, x_{2} \in Q, \\ \left.\frac{x_{1}+2 x_{2}}{3}, 1\right], & \text { if } x_{1}<x_{2}, x_{1} \notin Q, \\ \emptyset, & \text { or } x_{2} \notin Q, \\ \{0\}, & \text { if } x_{1}=x_{2}, \\ \text { if } x_{1}>x_{2} .\end{cases}
$$

Then $A \cap(H-\operatorname{co} P)=A \cap P$ is transfer open inverse valued in $X$. Indeed, $(A \cap P)^{-1}(0)=P^{-1}(0)=\left\{\left(x_{1}, x_{2}\right) \mid x_{1}>x_{2}\right\}$ is open in $X \times X$, and for any $t \in(0,1]$ and $\left(x_{1}, x_{2}\right) \in$ $(A \cap P)^{-1}(t)=P^{-1}(t)$, we always have $\left(x_{1}, x_{2}\right) \in \operatorname{int} P^{-1}(1)=$ $\operatorname{int}(A \cap P)^{-1}(1)$. However, the lower section $(A \cap P)^{-1}(1 / 2)$ is not open. Indeed, let $a_{n}=(1 / 2)-(1 / n \sqrt{2})$ and let $b_{n}=9 / 10$; then $\left(a_{n}, b_{n}\right) \in X \times X \backslash(A \cap P)^{-1}(1 / 2)$ and $\left(a_{n}, b_{n}\right)$ converges to $(1 / 2,9 / 10)$, which does not belong to $X \times X \backslash(A \cap P)^{-1}(1 / 2)$. This means that the set $X \times X \backslash(A \cap P)^{-1}(1 / 2)$ is not closed, and hence $(A \cap P)^{-1}(1 / 2)$ is not open. Further, for each $x_{1}, x_{2} \in$ $X, x_{1} \notin P\left(x_{1}, x_{2}\right)=H-\operatorname{co} P\left(x_{1}, x_{2}\right)$. Thus, all hypotheses of Corollary 9 are satisfied so that the generalized abstract economy $\Omega$ has an equilibrium point in $X \times X$. In fact, all the equilibria of $\Omega$ are the points $(a, a)$, where $a \in[0,1]$.

Let $X$ and $Y$ be two topological spaces. Given three setvalued mappings $T: X \rightarrow 2^{Y}, F: X \rightarrow 2^{X}, A: X \rightarrow 2^{X}$, 
and a function $\phi: X \times X \times Y \rightarrow \mathbb{R}$, a generalized quasivariational inequality is defined as follows:

$$
(\text { GQVI })\left\{\begin{array}{l}
\text { Find }(\widehat{x}, \widehat{w}, \widehat{y}) \in X \times X \times Y \\
\quad \text { such that } \widehat{x} \in \operatorname{cl} A(\widehat{x}), \widehat{w} \in F(\widehat{x}), \\
\quad \widehat{y} \in T(\widehat{x}), \\
\phi(z, \widehat{x}, \widehat{y}) \geq 0, \\
\quad \forall z \in A(\widehat{x}) \cap\left(F^{-1}(\widehat{w})\right)^{C} .
\end{array}\right.
$$

In particular, if $F(x)=\{x\}$ for each $x \in X$, then $\left(F^{-1}(w)\right)^{C}=\{w\}^{C}=X \backslash\{w\}$. Therefore, the (GQVI) reduces to the usual quasi-variational inequality as follows:

$$
(\mathbf{Q V I})\left\{\begin{array}{l}
\text { Find }(\hat{x}, \hat{y}) \in X \times Y \\
\quad \text { such that } \widehat{x} \in \operatorname{cl} A(\widehat{x}), \quad \widehat{y} \in T(\widehat{x}), \\
\phi(z, \hat{x}, \hat{y}) \geq 0, \quad \forall z \in A(\widehat{x}) \backslash\{\hat{x}\} .
\end{array}\right.
$$

Theorem 11. Let $(X, \Gamma)$ be an l.c.-space with precompact polytopes, $\Gamma_{\{x\}}=\{x\}$ for each $x \in X$, and let $Y$ be a topological space. The set-valued mappings $T: X \rightarrow 2^{Y}$ and $F, A: X \rightarrow$ $2^{X}$ satisfy $T \in H(X, Y), F \in H(X, X)$, and $\operatorname{cl} A \in H(X, X)$, and $A^{-1}(x)$ is open for all $x \in X$. Suppose that $\phi: X \times X \times Y \rightarrow$ $\mathbb{R}$ is a function such that

(1) $\phi_{\alpha}(x, x, y) \geq 0$ for all $x \in X$ and $y \in T(x)$,

(2) for each fixed $z \in X$, the mapping $(x, y) \mapsto \phi(z, x, y)$ is lower semicontinuous,

(3) for each fixed $(x, y) \in X \times Y$, the mapping $z \mapsto$ $\phi(z, x, y)$ is $H$-quasiconvex in the following sense that for any finite set $D$ in $X$,

$$
\phi(z, x, y) \leq \max _{u \in D} \phi(u, x, y), \quad \forall z \in H-\operatorname{co} D .
$$

\section{Then there is a solution to (GQVI).}

Proof. Define a set-valued mapping $P: X \times X \rightarrow 2^{X}$ by

$$
\begin{array}{r}
P(x, w):=\left\{z \in X \mid \inf _{y \in T(x)} \phi(z, x, y)<0\right\} \cap\left(F^{-1}(w)\right)^{C}, \\
\forall(x, w) \in X \times X .
\end{array}
$$

By [20, Proposition 23, page 121], for each fixed $z \in X$, the mapping $x \mapsto \inf _{y \in T(x)} \phi(z, x, y)$ is lower semicontinuous. Thus, the set $\left\{x \in X \mid \inf _{y \in T(x)} \phi(z, x, y)>0\right\}$ is open for each $z \in X$. It follows that

$$
\begin{aligned}
P^{-1}(z)= & \left(\left\{x \in X \mid \inf _{y \in T(x)} \phi(z, x, y)>0\right\} \times X\right) \\
& \cap\left(X \times(T(z))^{C}\right)
\end{aligned}
$$

is open. By Lemma $5,(H-\operatorname{co} P)^{-1}(z)$ is also open. Next, we show that $x \notin H-\operatorname{co} P(x, w)$ for all $x, w \in X$. Assume that there are $x_{0}$ and $w_{0}$ satisfying $x_{0} \in H-\operatorname{co} P\left(x_{0}, w_{0}\right)$. Then there is a finite subset $D$ of $P\left(x_{0}, w_{0}\right)$ such that $x_{0} \in H$-coD. For each fixed $y \in T\left(x_{0}\right)$, since the mapping $z \mapsto \phi\left(z, x_{0}, y\right)$ is $H$-quasiconvex, it follows that

$$
0 \leq \inf _{y \in T\left(x_{0}\right)} \phi\left(x_{0}, x_{0}, y\right) \leq \inf _{y \in T\left(x_{0}\right)} \max _{z \in D} \phi\left(z, x_{0}, y\right) .
$$

By Kneser's minimax theorem [21], together with $z \in$ $P\left(x_{0}, w_{0}\right)$ for all $z \in D$, we have

$$
\inf _{y \in T\left(x_{0}\right)} \max _{z \in D} \phi\left(z, x_{0}, y\right)=\max _{z \in D} \inf _{y \in T\left(x_{0}\right)} \phi\left(z, x_{0}, y\right)<0 .
$$

This is a contradiction. Thus, all hypotheses of Corollary 7 are satisfied. Therefore, there exist $\widehat{x}, \widehat{w} \in X$ such that $\widehat{x} \in$ $\operatorname{cl} A(\widehat{x}), \widehat{w} \in F(\widehat{x})$, and $A(\widehat{x}) \cap P(\widehat{x}, \widehat{w})=\emptyset$, It follows that

$$
\inf _{y \in T(\widehat{x})} \phi(z, \widehat{x}, y) \geq 0, \quad \forall z \in A(\widehat{x}) \cap\left(F^{-1}(\widehat{w})\right)^{C} .
$$

Since $T(\hat{x})$ is compact, there is $\hat{y} \in T(\hat{x})$ such that $\phi(z, \widehat{x}, \widehat{y}) \geq 0$ for all $z \in A(\widehat{x}) \cap\left(F^{-1}(\widehat{w})\right)^{C}$. That is, $(\widehat{x}, \widehat{w}, \widehat{y})$ is a solution to (GQVI).

\section{References}

[1] W. Shafer and H. Sonnenschein, "Equilibrium in abstract economies without ordered preferences," Journal of Mathematical Economics, vol. 2, no. 3, pp. 345-348, 1975.

[2] X. P. Ding, "Fixed points, minimax inequalities and equilibria of noncompact abstract economies," Taiwanese Journal of Mathematics, vol. 2, no. 1, pp. 25-55, 1998.

[3] X. P. Ding, W. K. Kim, and K.-K. Tan, "Equilibria of noncompact generalized games with $L^{*}$-majorized preference correspondences," Journal of Mathematical Analysis and Applications, vol. 164, no. 2, pp. 508-517, 1992.

[4] L. G. Epstein and S. Ji, "Ambiguous volatility and asset pricing in continuous time," CIRANO Working Papers 2012s-29, CIRANO, 2012.

[5] N. J. Huang, "The existence of equilibria for generalized abstract economics," Indian Journal of Pure and Applied Mathematics, vol. 29, no. 8, pp. 801-809, 1998.

[6] N. J. Huang, "Some new equilibrium theorems for abstract economies," Applied Mathematics Letters, vol. 11, no. 1, pp. 41$45,1998$.

[7] W. K. Kim and K.-K. Tan, "New existence theorems of equilibria and applications," Nonlinear Analysis: Theory, Methods and Applications, vol. 47, no. 1, pp. 531-542, 2001.

[8] L.-J. Lin, L.-F. Chen, and Q. H. Ansari, "Generalized abstract economy and systems of generalized vector quasi-equilibrium problems," Journal of Computational and Applied Mathematics, vol. 208, no. 2, pp. 341-353, 2007.

[9] Z.-F. Shen, "Maximal element theorems of $H$-majorized correspondence and existence of equilibrium for abstract economies," Journal of Mathematical Analysis and Applications, vol. 256, no. 1, pp. 67-79, 2001.

[10] S. Al-Homidan and Q. H. Ansari, "Fixed point theorems on product topological semilattice spaces, generalized abstract economies and systems of generalized vector quasi-equilibrium problems," Taiwanese Journal of Mathematics, vol. 15, no. 1, pp. 307-330, 2011.

[11] K.-K. Tan and X.-Z. Yuan, "Lower semicontinuity of multivalued mappings and equilibrium points," in World Congress of 
Nonlinear Analysts '92: Proceedings of the 1st World Congress of Nonlinear Analysts, vol. 8, pp. 1849-1860, Walter De Gruyter, Berlin, Germany, 1996.

[12] E. Tarafdar and P. J. Watson, "Coincidence and the FanGlicksberg fixed point theorem in locally $\mathrm{H}$-convex uniform spaces," Tech. Rep., The University of Queensland, 1997.

[13] X. Wu and Z.-F. Shen, "Equilibrium of abstract economy and generalized quasi-variational inequality in $H$-spaces," Topology and its Applications, vol. 153, no. 1, pp. 123-132, 2005.

[14] G. X.-Z. Yuan and E. Tarafdar, "Maximal elements and equilibria of generalized games for $U$-majorized and condensing correspondences," International Journal of Mathematics and Mathematical Sciences, vol. 22, no. 1, pp. 179-189, 1999.

[15] J. L. Kelley, General Topology, Springer, New York, NY, USA, 1975.

[16] Y.-L. Wu, C.-H. Huang, and L.-J. Chu, "An extension of Mehta theorem with applications," Journal of Mathematical Analysis and Applications, vol. 391, no. 2, pp. 489-495, 2012.

[17] C. D. Horvath, "Contractibility and generalized convexity," Journal of Mathematical Analysis and Applications, vol. 156, no. 2, pp. 341-357, 1991.

[18] N. C. Yannelis and N. D. Prabhakar, "Existence of maximal elements and equilibria in linear topological spaces," Journal of Mathematical Economics, vol. 12, no. 3, pp. 233-245, 1983.

[19] X. Wu and G. X.-Z. Yuan, "On equilibrium problem of abstract economy, generalized quasi-variational inequality, and an optimization problem in locally $H$-convex spaces," Journal of Mathematical Analysis and Applications, vol. 282, no. 2, pp. 495504, 2003.

[20] J.-P. Aubin and I. Ekeland, Applied Nonlinear Analysis, John Wiley \& Sons, New York, NY, USA, 1984.

[21] H. Kneser, "Sur un théorème fondamental de la théorie des jeux," Comptes Rendus de l'Académie des Sciences, vol. 234, pp. 2418-2420, 1952. 


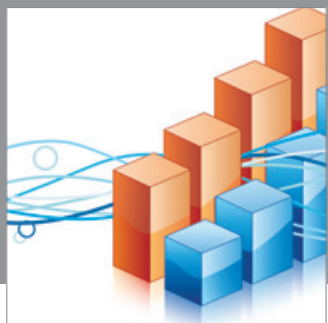

Advances in

Operations Research

mansans

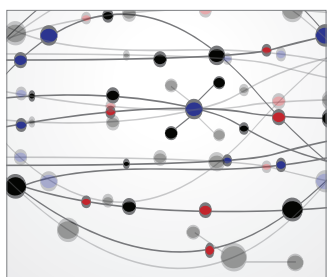

The Scientific World Journal
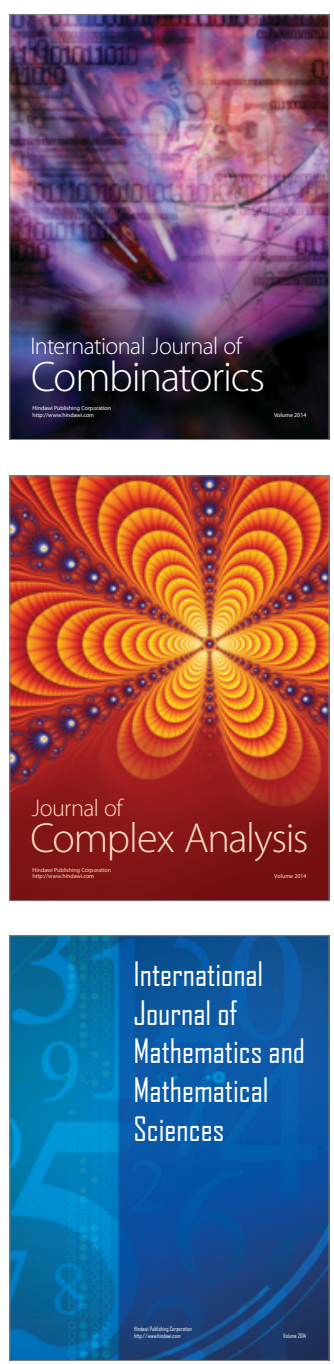
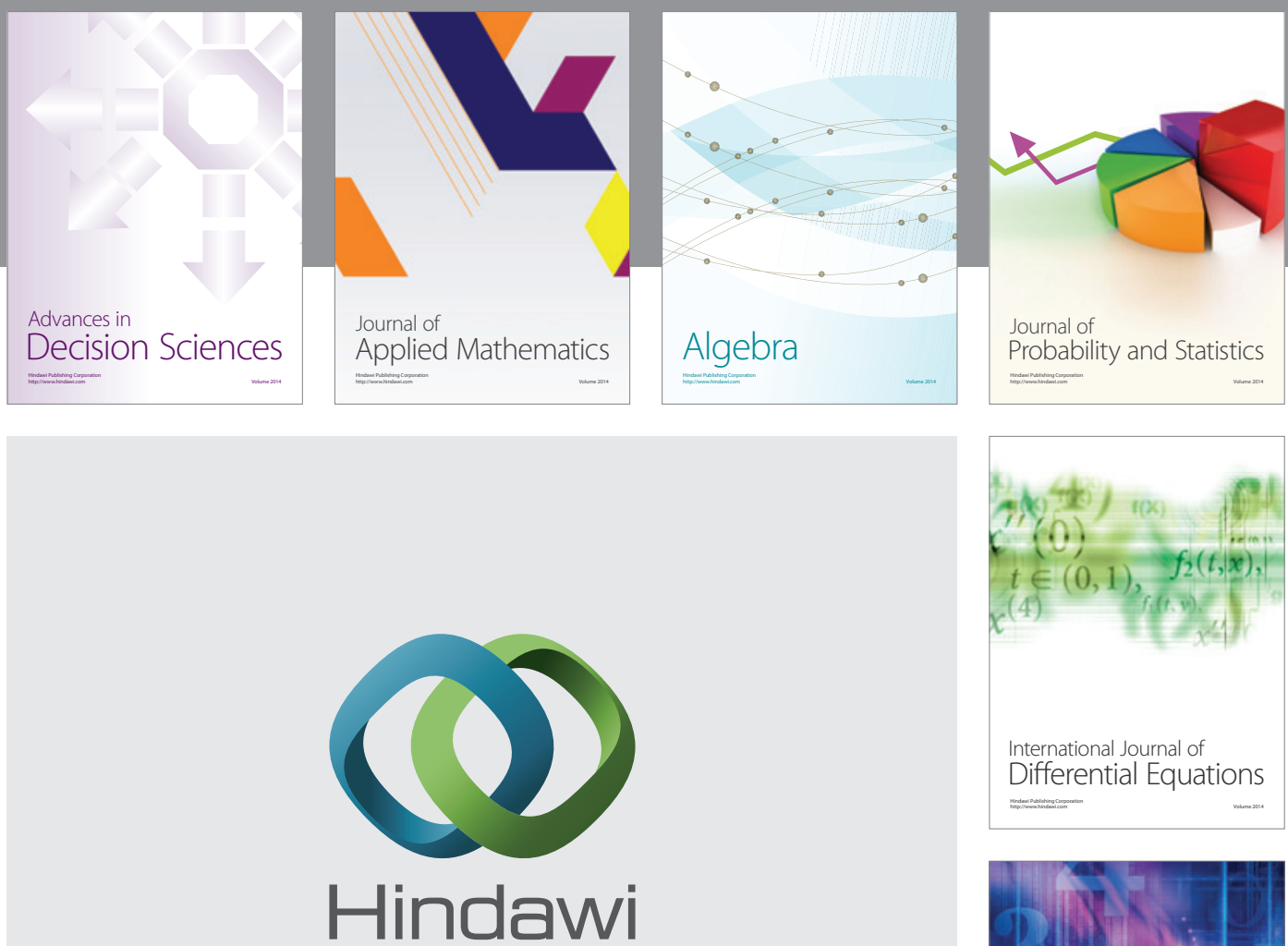

Submit your manuscripts at http://www.hindawi.com
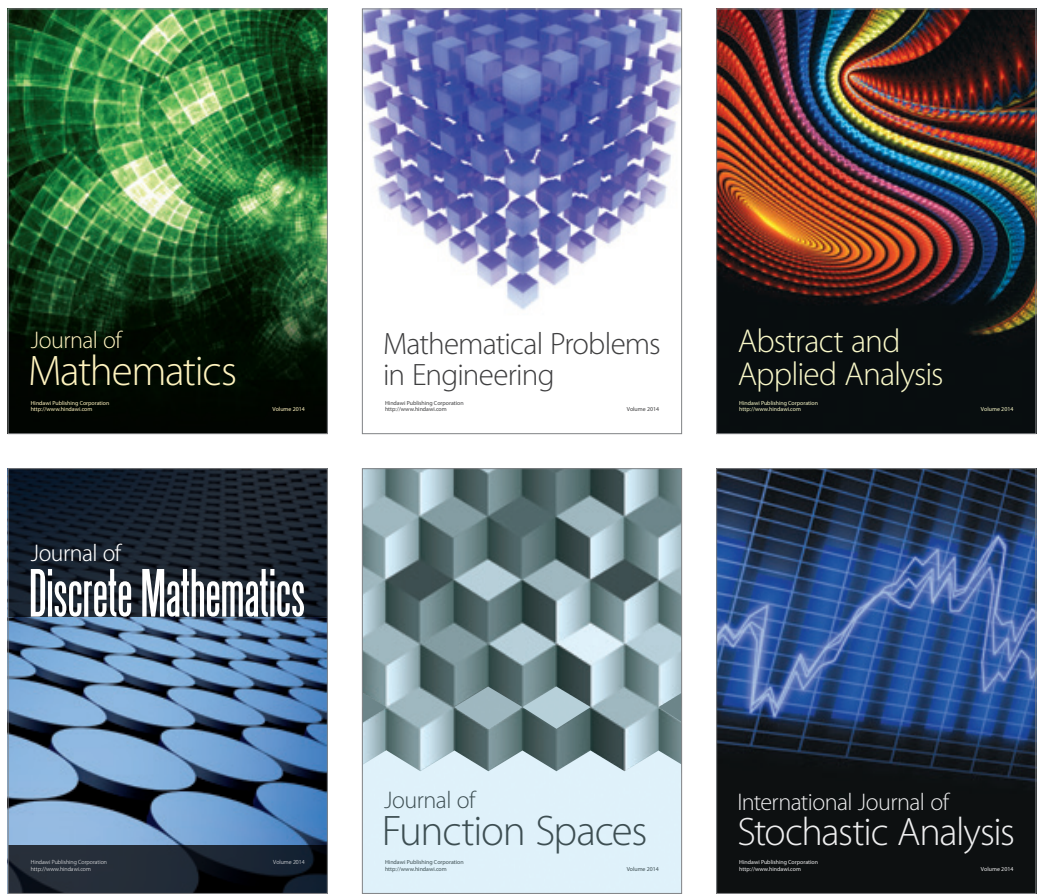

Journal of

Function Spaces

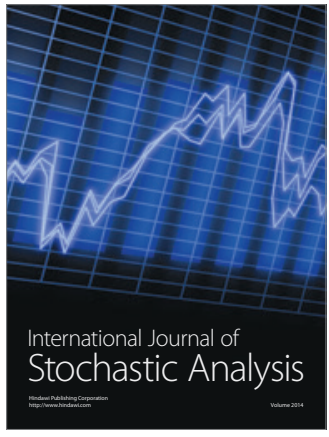

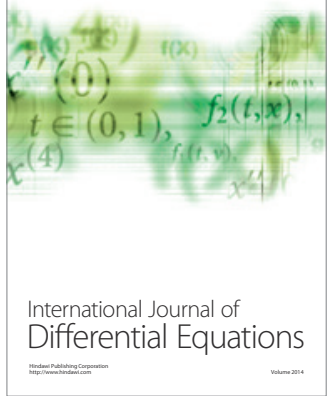
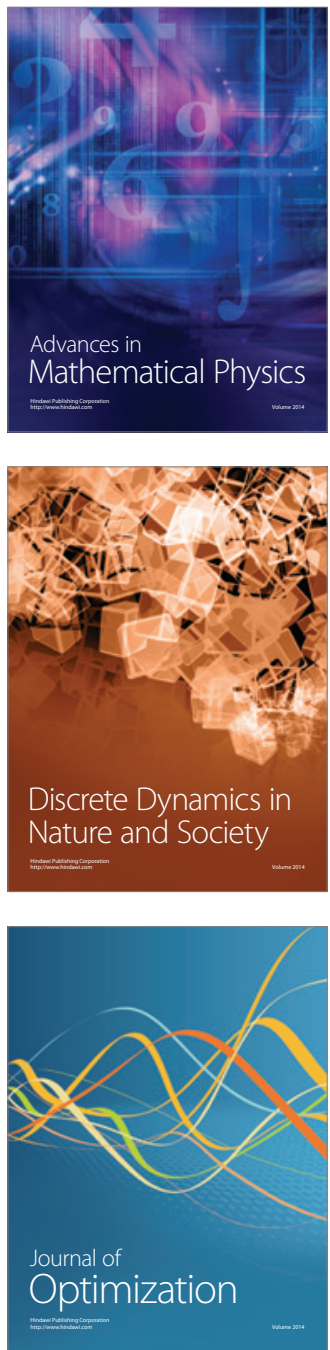